\title{
Response to the Kurdish refugee crisis by the Edinburgh MEDIC 1 team
}

\author{
T R J Parke, G Haddock, D J Steedman, A J Pollok, K Little
}

Department of Accident and Emergency Medicine, Royal Infirmary of Edinburgh, Edinburgh EH3 9YW

T R J Parke, MRCP, registrar D J Steedman, MD, consultant

$\mathrm{K}$ Little, $\mathrm{MD}$, consultant

Department of Surgical Paediatrics, Royal Hospital for Sick Children, Edinburgh EH9 1LF G Haddock, FRCS, registrar in surgical paediatrics

\section{Department of}

Anaesthetics, Royal

Infirmary of Edinburgh,

Edinburgh EH3 9YW

A J Pollok, FFARCS,

consultant anaesthetist

Correspondence to:

Dr Little.

BMF 1992; 304:695-7
In March 1991, because of heightened internal tensions in the aftermath of the Gulf war, about two million Kurds abandoned their homes and established makeshift camps in the mountains of northern Iraq. Owing to appalling weather and poor sanitation, casualties mounted, initiating an international relief effort. As part of the British response the accident and emergency department of the Royal Infirmary, Edinburgh, prepared a seven strong medical and nursing team to travel to northern Iraq and provide emergency medical aid to the displaced Kurds. The team was based on the MEDIC 1 emergency resuscitation flying squad ${ }^{1}$ and comprised two consultants, a registrar, and two senior nurses from the accident and emergency department supported by a consultant anaesthetist and a paediatric surgical registrar.

A plan for securing equipment and stores for an international response by the MEDIC 1 team had previously been established. This expedited the gathering of general and medical supplies, which were packed into 60 boxes weighing over two tons. Medical supplies were targeted at expected clinical conditions and assembled on the basis of treating 100 patients a day for two weeks. These comprised intravenous fluids, dressings and casting material, drugs (mainly antibiotics and analgesics), several trays of surgical instruments, and a "Triservice" field anaesthetic machine. General equipment included clothing for extremes of weather, tents, and enough food and water for two weeks. Funding for the equipment, locum cover, and travel was provided by the Overseas Development Administration and the European Community.

On 5 May, three weeks after preparations had been completed, the MEDIC 1 team was flown to Incirlik airbase in Turkey as part of a group of 90 civilian volunteers assembled by the Overseas Development Administration. This group, composed of health care workers, engineers, and fire service personnel, was then driven by bus 500 miles east to a military base at Silopi on the Turkey-Iraq border, where the volunteers were divided into seven multidisciplinary "mobile support teams." These were to operate throughout the safe haven area of northern Iraq under the protection of the Allied Forces, providing basic health care, sanitation, clean water supplies, and food distribution networks. The MEDIC 1 team formed the core of the mobile support team sent to the town of Zakhu to help provide medical services in both the local hospital and the rapidly expanding refugee camp. The team was augmented by a general practitioner, four nurses, and four firemen from the Greater Manchester fire brigade.

\section{Zakhu camp}

The Zakhu refugee camp contained 30000 recent arrivals from the mountains, located both in a formal camp with adequate facilities and in a prescreening area that lacked basic amenities, including any form of health care. There were two jobs for the team in the Zakhu camp. Firstly, we had to manage acute medical conditions arising in the prescreening area, and, secondly, we had to undertake the immunisation and health screening required before people were admitted to the formal camp.

The prescreening camp was hot, insanitary, and chaotic. This area accommodated the most recent arrivals from the squalid mountain camps and had a transient population of about 5000 refugees, many of whom were acutely ill. There were large numbers of children, despite the fact that many had died in the mountains at the start of the crisis. The firemen constructed a field hospital to an agreed design in this informal camp. Five military general purpose medium tents were used, with heavy duty plastic sheeting used for flooring and partitions. The hospital was furnished with army campbeds and chipboard tables constructed on site. It had two wards (one ward would be occupied as the other was cleaned daily), an outpatient department, an emergency room, a kitchen, a pharmacy, and a large store.

Within five minutes of opening the field hospital was inundated with patients, either self referred or brought directly by medivac helicopter from the mountains. The field hospital was operational for eight days, and most of the workload was related to infectious diseases in young adults and infants (see box).

The younger children often presented with dehydration secondary to prolonged simple diarrhoea though some had symptoms suggestive of dysentery. These children were treated with premixed oral rehydration solution, the first calculated quantity of which was administered in the outpatient department under supervision. More severely affected children
^One box of records containing about 100 casesheets was mislaid during evacuation and these data are excluded. 
(5-10\% of body weight lost) or those not tolerating oral administration were admitted for nasogastric rehydration with oral rehydration solution, and those most severely affected (more than $10 \%$ body weight lost) were admitted and rehydrated with intravenous one fifth strength saline-dextrose. The infusions required constant supervision as anxious mothers, believing that more must be better, altered the flow rates. Antibiotic treatment was considered for persistent diarrhoea or in the presence of systemic upset. Smaller numbers of older children had evidence of proteinenergy malnutrition or pellagra, or both, shown by dry cracked skin, ankle oedema, weight loss, and apathy. Infant formula milk was the most readily available feed and was administered (via the nasogastric route when necessary) along with vitamin supplementation.

A distinct group of young adults (14-25 years) presented with a prodromal illness of 7-10 days' duration with cough, anorexia, constipation, and vague abdominal pains. They then developed a high fever of $39 \cdot 5-41^{\circ} \mathrm{C}$ with rigors, hypotension, and diarrhoea. We presumed this to be typhoid and treated patients with chloramphenicol combined with aggressive intravenous fluid resuscitation. The fever abated and wellbeing and appetite returned after four to five days, but not before several further spikes of high temperature and profound episodes of hypotension occurred.

Apart from scorpion stings and road traffic accidents, most of the trauma consisted of older infected wounds sustained in the mountains, such as compound fractures, bullet wounds, and cold injury. These usually required debridement and antibiotics.

The screening centre was run by one doctor and two nurses, who would see 500-1000 new attendances a day. Young children were given measles vaccine and vitamin A. Families were issued with a ticket for a tent, and were given a full health check. People who arrived sick were referred to the field hospital for further management. Abandoned children were unusual, though interim care was necessary for one 3 month old infant who had been found by a medivac team in the mountains.

\section{Zakhu hospital}

Medical services at Zakhu hospital were being coordinated by the Dutch army, helped by civilian organisations. The only surgical input, which had been provided by a French military unit, had been withdrawn just before our arrival. Therefore it was decided to supply an operating team to the hospital consisting

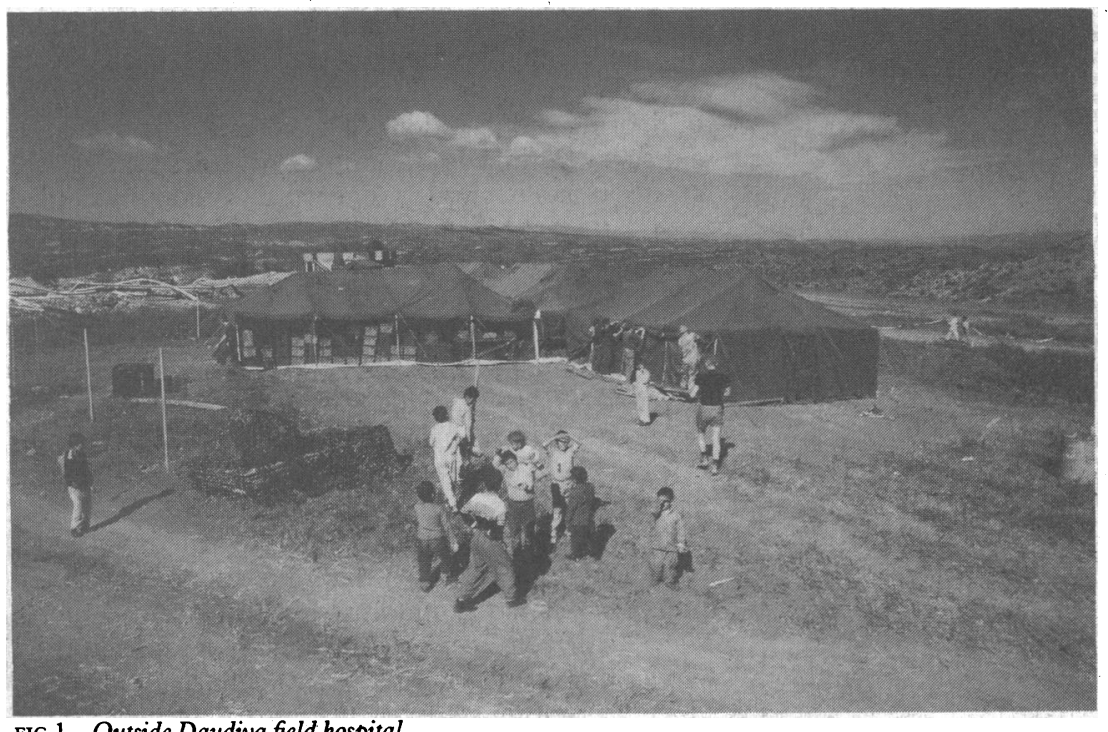

FIG 1-Outside Daudiya field hospital of one surgeon, one anaesthetist, and one fireman, who acted as scrub nurse and anaesthetic assistant. All surgical beds (together with mattresses on the ward floor and stretchers in the corridors) were occupied by patients in need of, or having just had, surgery. The operating theatre needed repairs and thorough cleaning, and serious deficiencies in both surgical instruments and anaesthetic drugs required supplementation from our supplies.

On the first day three elderly women were identified who required leg amputations for gangrene secondary to frostbite. In the following eight days 19 major and 15 minor operations were performed in 25 patients, 15 of whom were aged under 16 years.

On the evening of the fourth day the first of six bomb victims was admitted. An 8 year old boy had had his right hand and the right side of his face blown off while playing with an unexploded grenade and had suffered severe penetrating injuries to both eyes. He required a mid-forearm amputation, reconstruction of his face, and removal of shrapnel from his chest and abdomen. In the following four days five other young boys suffered similar blast injuries, necessitating partial hand amputation in four and debridement of a severely comminuted fractured upper tibia and a compound depressed skull fracture in one. Two of these patients required postoperative ventilation, a facility not available at the hospital. They were evacuated by helicopter to a hospital 100 miles north west at Dyarbakir in Turkey.

In addition to the trauma victims the team encountered many patients with advanced surgical pathology, including tumours, malunited fractures, and infected open wounds. The surgical management of these was thought to be outside the scope of the available resources and reluctantly we made the difficult decision to do nothing.

By 17 May the need for an acute medical centre in the prescreening area had diminished as people dispersed into the formal camps or continued onwards to their homes. In addition Iraqi medical and nursing staff were returning to the hospital and beginning to take over from the foreign relief teams. After discussion with the Overseas Development Administration the team redeployed on 20 May to provide a field hospital in the camp at Daudiya, 30 miles to the south east of Zakhu.

\section{Daudiya}

A small refugee camp of 6000 people had been established in the ruined village of Daudiya. An American military medical unit was already on site providing inpatient and outpatient services. Four to five miles away the British Marine medical squadron was located in Sirsenk fully equipped for surgery. The task was to help both the American and British military units to provide health care as the population increased in the camp and to establish a civilian medical presence in this sector. A field hospital was again designed and constructed from five general purpose medium tents. From experience gained in Zakhu, the firemen had the hospital erected and working within 24 hours, and several minor design improvements were incorporated into the layout plan.

The nature of the work was very similar to that at Zakhu if a little less frenetic (see box). There were fewer dehydrated and malnourished infants but they were just as severely ill on presentation. The typhoid cases continued but, gratifyingly, responded to the treatment regimen developed in Zakhu. More unusual conditions were also encountered, such as nephrotic syndrome and juvenile polyarthritis.

Supplies were readily available from the Sirsenk airbase, and adequate feeding materials were more easily 


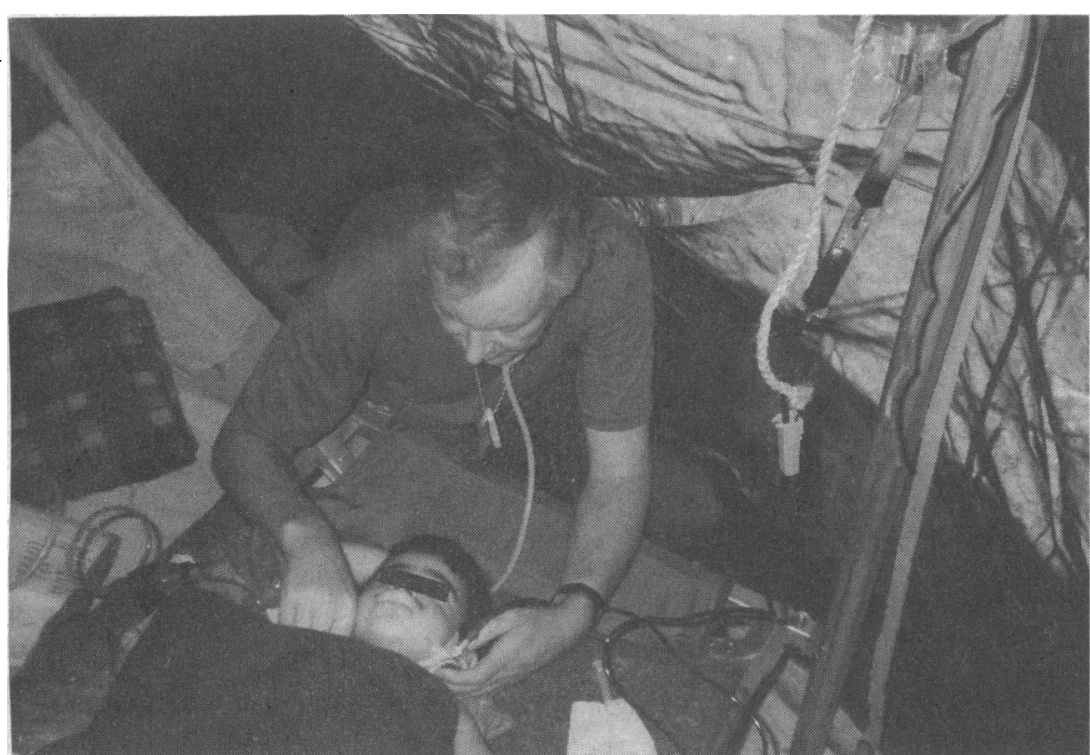

FIG 2-Resuscitation in Daudiya field hospital obtained. Children requiring urgent feeding, either orally or by nasogastric tube, were given augmented formula milk if less than 3 months old and high energy milk made from dried skimmed milk, oil, and sugar if more than 3 months old. Two to three days was the maximum inpatient stay tolerated by the parents, and rehydration and feeding had to be concentrated into this short timescale. Although many of the malnourished children thus treated had not regained their ideal weight on discharge, the intensive rehydration and feeding seemed to be able to reduce their lethargy such that they would take nourishment again.

There was a steady stream of minor and moderate injuries treated at the field hospital, and surgical help was provided to the medical squadron when seven multiply injured children presented after a landmine explosion nearby.

The expected rapid expansion of the camp at Daudiya did not occur. After the liberation of the large town of Dihok on the southern boundary of the safe haven many of the displaced Kurds began returning direct to their homes from the mountains. On 30 May the hospital was handed over to medical and nursing staff from a second group of Overseas Development Administration relief workers recently arrived from the United Kingdom. This group included three Kurdish doctors. A few hours later the MEDIC 1 team was evacuated by the French airforce to Incirlik, where the original group of volunteers reassembled.

On 3 June, four weeks after mobilising, the MEDIC 1 team returned home to Edinburgh. Despite the ever present danger of unexploded mines, the team suffered no serious casualties, though there were five cases of dysentery, one case of cellulitis, two chest infections, a urinary tract infection, and one laceration requiring suturing. Supplies of food and water were plentiful, if unpalatable, and members of the team lived in their own tents throughout.

\section{Conclusions}

After a disaster the requirements for medical aid vary from situation to situation, but there is a relatively predictable pattern. ${ }^{2-4}$ Initially there are large numbers of casualties at the site of the disaster. These overwhelm any existing local facilities and so salvagable patients may die. After several days to weeks the destruction of local infrastructure may lead to a second wave of casualties from infectious diseases, dehydration, and malnutrition, to which the very old and very young are particularly susceptible. In the final phase there is a prolonged period of reconstruction. In Iraq the delay in mobilising medical teams meant that first phase casualties died from hypothermia and trauma. ${ }^{5}$ During the second phase the medical workload consisted mainly of trauma and infectious diseases, and many patients were successfully treated with simple but intensive measures (such as surgical wound toilet, rehydration therapy, and appropriate antibiotics). The broad skill mix and accident and emergency base of the MEDIC 1 team allowed considerable flexibility in treating such a mixture of conditions, and prior experience of working together made for a social cohesion that supported individuals through difficult periods. Undoubtedly, the effectiveness of our response was enhanced by the addition of the extra nurses and the firemen.

The Overseas Development Administration concept of civilian mobile support teams dispatched to help in this disaster was inspired, courageous, and effective. Rather than relying on randomly grouping unscreened volunteers at the site of the disaster, however, the administration should maintain a register of teams with accident and emergency skills who can respond quickly and call on additional specialist input as appropriate to the particular incident. If and when the next international disaster occurs Britain needs rapidly to mobilise such teams within days to start first and second phase health care and to assess the requirement for follow up medium to long term medical aid.

The MEDIC 1 team consisted of the authors together with Sister Margaret White and Staff Nurse Fiona Robertson. We gratefully acknowledge the help of Dr C E Robertson, Mrs E MacDonald, and the staff of the Royal Infirmary in organising this mission and Mrs E Laing for her help in preparing the manuscript.

1 Dark P, Little K, Steedman D, Gordon M, Robertson C. Objective analysis of an accident flying squad. Scot Med f 1990;35:73-6.

2 Rutherford W. Major disasters abroad. Injury 1990;21:15-6.

3 Redmond AD. Response of the SMART team to the earthquake in Armenia and the Lockerbie air disaster. BMF 1989;299:611-2.

4 Redmond AD. The South Manchester accident rescue team and the earthquak in Iran, June 1990. BMf 1991;302:1521-3.

5 Guild R. Crisis in the help service. Guardian 1991;8 May:p19.

(Accepted 29 September 1991 )

of some vaccines. If the buttock is used care should be taken to make the injection in its outer/upper quadrant well away from the sciatic nerve. Intradermal injections (BCG vaccine) are given in the upper arm but slightly distally to the deeper injections. The injection is done ove the insertion of the deltoid. ${ }^{2}$ - ANGUS NICOLL, consultant PHLS Communicable Disease Surveillance Centre

1 Joint Committee on Vaccination and Immunisation. Immunisation against infectious disease. London: HMSO, 1990.

2 Rudd $\mathrm{P}$, Nicoll A, eds. Manual on infections and immunisations in children. Oxford: British Paediatric Association, 1991:154-8. 